(C) 1977 IEEE. Personal use of this material is permitted. However, permission to reprint/republish this material for advertising or promotional purposes or for creating new collective works for resale or redistribution to servers or lists, or to reuse any copyrighted component of this work in other works must be obtained from the IEEE.

IEEE Transactions on Nuclear Science, Vol.NS-24, No.3, June 1977

\title{
A NEW MULTIDISCIPLINARY SEPARATED-SECTOR CYCLOTRON FACILITY
}

\author{
A.H. Botha, G.F. Burdzik, H. Dekker, G. Heymann, H.N. Jungwirth, S. Schneider \\ National Accelerator Project, CSIR, Pretoria
}

\author{
J.C. Cornel1, J.J. Kritzinger, F.J. Mumford
}

National Accelerator Project, SUNI, Faure

P.J. Celliers, Z.B. du Toit

Dept. Physics, Stellenbosch University, South Africa.

\section{Summary}

A separated-sector cyclotron with a maximum proton energy of $200 \mathrm{MeV}$ has been proposed for a multidisciplinary accelerator facility for the Republic of South Africa. At energies up to $100 \mathrm{MeV}$, proton and deuteron currents of the order of 100 microamps will be available for routine isotope production and neutron therapy. Light-ion beams for particle physics and chemistry will be delivered with maximum currents of about 10 microamps and maximum energies in excess of $50 \mathrm{MeV}$ per nucleon. Heavy-ion beams of between 15 and $50 \mathrm{MeV}$ per nucleon are planned with maximum intensities of between 10 particle-nanoamps and 1 particle-microamp, depending on the type of ion. This proposal has recently been approved and progress in the design of the cyclotron and related aspects of the proposed facility is reported here.

\section{Introduction}

A feasibility study of a multidisciplinary national accelerator facility for South Africa resulted in the recommendation of a separated-sector cyclotron (SSC) with a maximum $\mathrm{k}$-value of $200 \mathrm{MeV}$ as the machine most suited to the requirements of medicine (particularly radiotherapy), nuclear physics, nuclear chemistry and isotope production. 1 This recommendation was approved in principle towards the end of 1975.

Limited funds were then made available during 1976 during which a technical study was made to determine the characteristics and limitations of the proposed accelerator complex. Critical components and phenomena were studied by calculation and by measurements on scale models where necessary. The results of the study are summarised in a Technical Report. ${ }^{2}$

Funds for the 1977/78 financial year have been made available, and the project is now moving into the final design stage.

Although the location of the accelerator complex has not yet been selected, a number of possible sites in the vicinity of the major teaching hospitals and universities in the western cape Province have been evaluated. The more promising sites have been subjected to extensive subsoil investigation.

\section{General Description}

The proposed facility consists of a separatedsector cyclotron with four $34^{\circ}$ sector magnets and two delta resonators in opposite valleys. Flat-topping resonators will probably be added in the remaining valleys at a future date.

The beam requirements for the various fields of application are fairly diverse, ranging from high intensity proton and deuteron beams at medium energies for both isotope production and radiotherapy purposes, to very high quality beams at all energies for nuclear physics, chemistry and allied disciplines. This wide spectrum of particle type and energy can best be achieved by making use of two solid-pole cyclotrons as injectors, one (SPC1) for lighter ions and a second with an appreciably higher $k$-value for heavy ions. The main parameters of the three proposed cyclotrons are summarised in Table 1.

\begin{tabular}{|c|c|c|}
\hline The SSC & $\begin{array}{l}\text { K-value } \\
\text { Injection Radius } \\
\text { Extraction Radius } \\
\text { Largest Diameter } \\
\text { Magnetic Flux Density } \\
\text { Magnet Sectors } \\
\text { Magnet Pole Gap } \\
\text { Total Iron mass } \\
\text { Resonators } \\
\text { Delta Voltage } \\
\text { Rf Power Consumption } \\
\text { Frequency Range } \\
\text { Frequency Variation } \\
\text { Harmonic Numbers } \\
\text { Power Amplifier }\end{array}$ & $\begin{array}{l}200 \mathrm{MeV} \\
1,01 \mathrm{~m} \\
4,43 \mathrm{~m} \\
13,2 \mathrm{~m} \\
1,26 \mathrm{~T} \text { (max) } \\
4 \times 34^{\circ} \\
60 \mathrm{~mm} \\
1380 \mathrm{t} \\
2 \times 49^{\circ} \text { deltas, } \mathrm{N} / 2 \\
250 \mathrm{kV} \text { (max) } \\
<70 \mathrm{~kW} / \text { resonator } \\
5-27,5 \mathrm{MHz} \\
\mathrm{L} \& \mathrm{C} \\
4 \& 12 \\
130 \mathrm{~kW}\end{array}$ \\
\hline SPC1 & $\begin{array}{l}\text { K-value } \\
\text { Extraction Radius } \\
\text { Magnetic Flux Density } \\
\text { Resonators } \\
\text { Frequency Range } \\
\text { Harmonic Numbers }\end{array}$ & $\begin{array}{l}8 \mathrm{MeV} \\
47,6 \mathrm{~cm} \\
0,86 \mathrm{~T} \text { (max) } \\
2 \times 90^{\circ} \text { dees, } \lambda / 4 \\
5-27,5 \mathrm{MHz} \\
2 \& 6\end{array}$ \\
\hline SPC2 & $\begin{array}{l}\text { K-value } \\
\text { Extraction Radius } \\
\text { Magnetic Flux Density } \\
\text { Resonators } \\
\text { Frequency Range } \\
\text { Harmonic Numbers }\end{array}$ & $\begin{array}{l}40 \mathrm{MeV} \\
71,4 \mathrm{~cm} \\
1,293 \mathrm{~T} \text { (max) } \\
2 \times 180^{\circ} \text { dees, } \lambda / 4 \\
5-27,5 \mathrm{MHz} \\
3 \& 9\end{array}$ \\
\hline
\end{tabular}

The Separated-Sector Cyclotron

\section{Orbit Dynamics}

We have analytically investigated equilibrium orbit properties (with a stepped-edge magnetic field), spacecharge effects, transit-time effects at an accelerating gap and conditions for single-turn extraction. A phase expansion/compression effect due to the radial component of the rf electric field has not shown up in our numerical calculations. ${ }^{3}$ Computational developments include programs for processing and isochronizing measured magnetic fields and for calculating equilibrium orbits and orbits with acceleration.

\section{Magnets}

The design characteristics of the four SSC-magnets with a sector angle of $34^{\circ}$ have been reported elsewhere, 2,4 Initially the magnets will provide isochronous fields up to $1,3 \mathrm{~T}$ at extraction with a maximum power consumption of $700 \mathrm{~kW}$ for a 11 coils. Extension of the excitation rance by $20 \%$ using additional coils around the yokes is envisaged at a later stage in order to increase the heavy ion capabilities of the SSC.

\section{Rf-system}

The main components of the $r f-s y s t e m$ are two $\lambda / 2$ delta-resonators tunable from 5 to $27,5 \mathrm{Mhz}, 2,5$ Frequency variation will be accomplished with two variable shorts and six variable capacitors per resonator. The power consumption per resonator will be less than $70 \mathrm{~kW}$ at $27,5 \mathrm{MHz}$ for a dee-voltage of $250 \mathrm{kV}$. The dee voltage at extraction will be approximately three times larger than that expected at injection. 


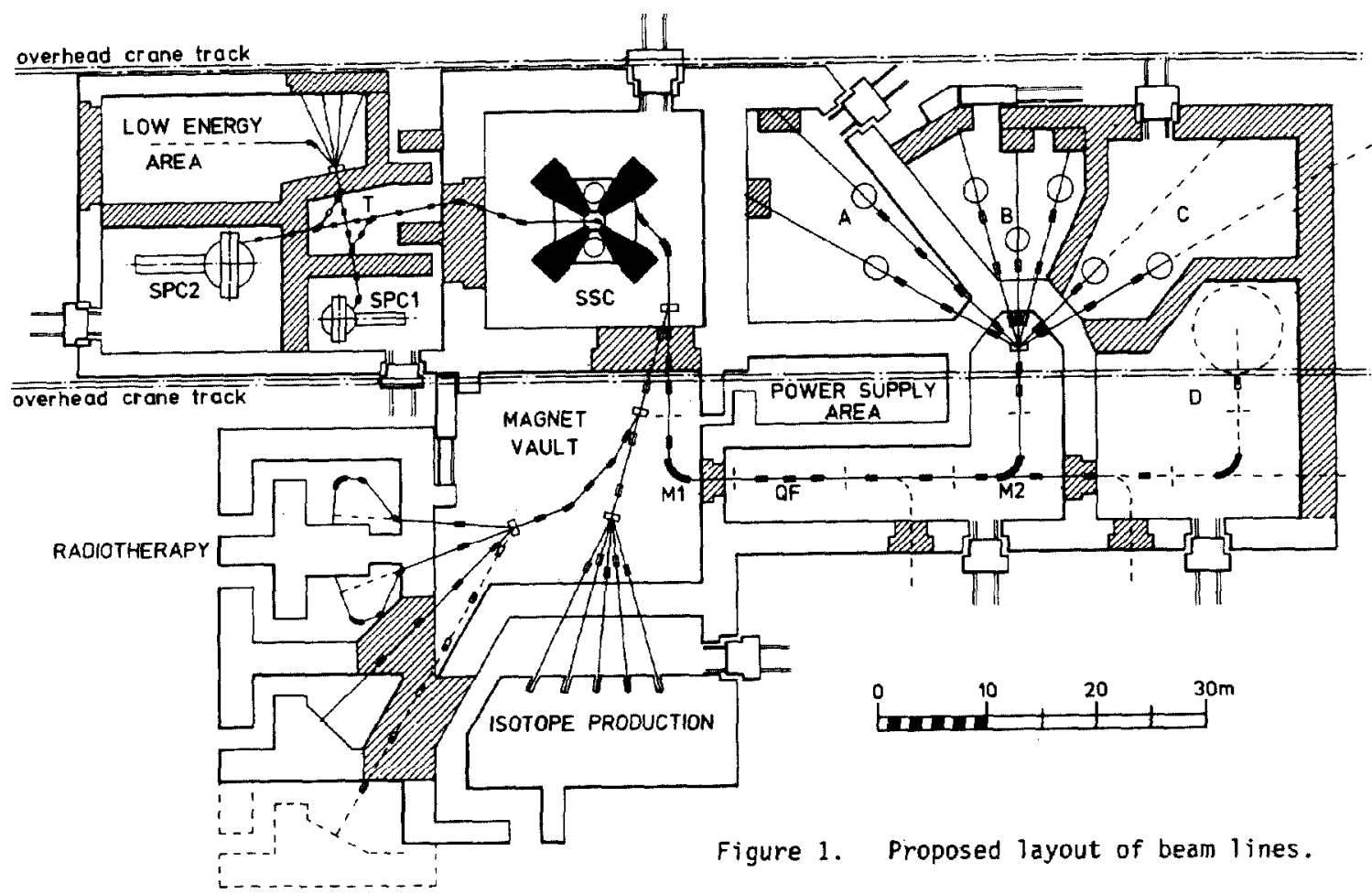

Vacuum

The cyclotron vacuum chamber will consist of 8 main sections: 2 delta resonators, 2 valley chambers and a chamber positioned in each of the 4 magnet pole gaps. The restriction imposed by the $60 \mathrm{~mm}$ pole gap on the thickness of vacuum chamber walls has led to two design proposals for this region which are currently being investigated. One possibility is a single thinwalled vacuum chamber inserted into the pole gap between the upper and lower trim coils and attached to the pole faces by a number of supports. The alternative involves a double vacuum chamber, but reduces the number of supports needed for the inner chamber.

Metal gaskets will be used wherever possible. The total chamber surface area will be about $300 \mathrm{~m}^{2}$ and the volume about $50 \mathrm{~m}^{3}$. The total gas load (without baking) due to outgassing, permeation and leaks is estimated to be of the order of $1 \times 10^{-2}$ torr litres per second after 15 hours of pumping.

It has been estimated that an operating pressure of $2 \times 10^{-7}$ torr in the median plane of the cyclotron will be needed to limit beam losses due to chargeexchange to less than 30 percent in the case of xenon, the heaviest ion considered.

A pumping speed of about $1 \times 10^{5}$ litres per secand will be required to maintain this operating pressure. Cryopumps and titanium-sublimation pumps are being considered as the main pumps for the major gas loads in the high vacuum regime. Both types provide high pumping capacity and do not contaminate the vacuum chamber with heavy hydrocarbons which enhance multipactoring of rf systems.

\section{Injection and extraction}

The beam (with maximum proton energy of $8 \mathrm{MeV}$ ) will be injected radially through a nearly field-free valley into the central region. A steerer magnet will direct particles into a 1 Tesla bending magnet followed by a 1,6 T magnetic inflector channel (in the pole-tip of a sector magnet) which will guide the beam onto the first well-centred orbit. Fine adjustments can be made with an electrostatic inflector channel in the next free valley.
Extraction can be accomplished wi thout making use of orbit resonances or field perturbations. For $200 \mathrm{MeV}$ protons the beam separation will be about $1 \mathrm{~mm}$. An initial electrostatic extraction channel, with a $0,1 \mathrm{~mm}$ thick pre-septum and a maximum field strength of 100 $\mathrm{kV} / \mathrm{Cm}$, will increase the orbit separation so that the current-carrying conductors of a septum magnet can be inserted in a succeeding valley. Finally a bending magnet will deflect the beam into the beam transport line. Flat-topping resonators will enhance the extraction dramatically.

\section{Beam diagnostics}

The emittance, intensity and energy of the preaccelerated beam will be measured in the transfer beam line before injection. In the central region, position probes in front of deflecting devices will locate the beam while a profile monitor will determine the spatial beam distribution. Three radial differential probes which can extend over the first third of the radial beam range will be used to centre the beam, to obtain radial beam information and to measure the beam intensity. Two radial beam probes, one before the electrostatic channel and one before the septum magnet will locate the beam at extraction. A multi-finger probe will determine the axial beam motion but will also be used to measure the beam intensity over the outer twothirds of the radial range. Non-interceptive phase probes will also be installed. Temperature sensors will be located at various critical positions, e.g., at septa. TV cameras can also be used for remote viewing of critical components or quartz viewer plates.

\section{Injectors}

A small reliable solid-pole cyclotron (SPC1) will initially be used as an injector for both light and heavy ions without stripping between the two machines. The proton beam energy of this machine will be $8 \mathrm{MeV}$. A second solid-pole cyclotron with a k-factor of 40 (SPC2) will probably be built at a later stage of the project as an injector for heavy ions with a stripper between the two machines. Energies attainable are indicated in Table 2 by calculated values for some representative ions. 
Table 2 Maximum Energies (MeV/nucleon)

\begin{tabular}{|cc|ccc|}
\hline \multicolumn{2}{|c|}{ SPCI + SSC } & \multicolumn{3}{|c|}{ SPC2 + SSC } \\
\hline Ion & $E_{\max }$ & Ion & Stripped & $E_{\max }$ \\
\hline$p$ & 200 & $14 \mathrm{~N}^{+}$ & $7^{+}$ & 37,4 \\
$\mathrm{~d}$ & 40,4 & $20 \mathrm{Ne}^{4^{+}}$ & $9^{+}$ & 32,4 \\
$3 \mathrm{He}^{2+}$ & 75,6 & $20 \mathrm{Ne}^{3+}$ & $8^{+}$ & 17,9 \\
$4 \mathrm{He}^{2+}$ & 40,4 & $40 \mathrm{Ar}^{6+}$ & $13^{+}$ & 16,5 \\
$14 \mathrm{~N}^{3}$ & 7,1 & $84 \mathrm{Kr}^{+}$ & $12^{+}$ & 3,1 \\
$20 \mathrm{Ne}^{3+}$ & 3,5 & $84 \mathrm{Kr}^{1} 1^{+}$ & $12^{+}$ & 2,6 \\
$40 \mathrm{Ar}^{+}$ & 2,4 & $132 \mathrm{Ke}^{11^{+}}$ & $13^{+}$ & 1,5 \\
\hline
\end{tabular}

Beam Transport

\section{Philosophy}

We have tried to separate such functions as momentum selection or phase-space matching from simple beam transportation for which achromatic and doubly telescopic sub-units can be used. The latter can consist of pairs of symmetric quadrupole triplets which can then be set up to give unit magnification. The symmetries of such systems reduce the number of working parameters to a minimum, and simplify the transport matrices by setting many of the elements to zero in both first and second order. The resulting system should then be relatively simple to operate and reasonably free from aberrations.

Transfer beam line

In coupling the two proposed injectors to the SSC, we require that either SPC1 or SPC2 can be used as an injector, while the beam from the other solid-pole cyclotron can simultaneously be directed to a lowenergy experimental area. A possible solution (indicated in area $T$ of figure 1), with a minimum number of quadrupoles has been designed with the aid of the program TRANSPORT: every path through this system is mirror symmetric, leading to doubly telescopic achromatic transfer with unit magnification.

Matching of the injector beams to the above system and matching of the bunched, stripped and analysed beam to the SSC have only been investigated in a preliminary way and detailed work is still in progress.

High intensity 1 ines

The beam extracted from the cyclotron will be corrected by a pair of quadrupoles and a dipole so that both dispersion and angular dispersion are zero. After being shaped, the beam will be deflected from the high resolution 1 ine and transported by double-telescopic triplet pairs to the isotope production switching magnet, or by an achromatic bend to the radiotherapy switching magnet.

\section{High resolution lines}

The momentum analysis for the nuclear science beam lines will be achieved by means of a double monochromator consisting of two $90^{\circ}$ dipole magnets with a symmetrizing insert of quadrupole triplets. 2 The properties of the system derive from its inherent symmetry, providing beam transport which can be either double-dispersive with a resolving power of 8000 for a $1 \mathrm{~mm}$ slit width ( $\triangle \mathrm{E}=25 \mathrm{keV}$ at $200 \mathrm{MeV}$ ), or achromatic and exactly double-telescopic to first order. By slight relaxation of the double-telescopic condition it is possible to compensate for the lengthening of the beam packets during transportation to the various target areas.

Switching of the beam beyond the double monochromator is best done by dipole magnets with circular pole pieces placed at a double waist. The beam can then be switched through $45^{\circ}$ and more with zero dispersion and littie angular dispersion.

\section{Control system}

The Instrumentation Division of the Atomic Energy Board has designed a computer-based control system for the accelerator complex. ${ }^{6}$ The proposed system consists of a multi-minicomputer system with CAMAC interfacing between computer and instrumentation. Certain aspects of hardware already completed for other systems using CAMAC are directly applicable to the present project. A software system based on existing program modules is suggested. A modular man-machine communication system, based on CAMAC and used in numerous systems elsewhere is proposed. The envisaged system has a hierarchical structure, with two senior-level computers (main and auxilliary) and three junior level computers. If the senior level computers fail, a limited form of control ("graceful degradation") can still be exercised by the junior computers. With certain automatic monitoring tasks performed by equipment already developed and with sufficient replacement equipment, a system with high reliability and minimum down-time should be achieved.

\section{Cooling system}

The peak power consumption of the facility will be 4Mw. There are unfortunately no natural sources of cooling water available to carry away this amount of heat. A maximum wet bulb temperature of $35^{\circ}$ preciudes cooling with cooling towers alone. It is therefore imperative to cool the facility with refrigerated water. In order to increase the reliability of the system two interchangeable cooling towers and refrigerators will be used.

\section{Buildings}

The building layout is greatly influenced by the directions of the various beamlines to and from the $200 \mathrm{MeV}$ cyclotron. The SSC and injector vaults together with the nuclear science target areas and associated shielding walls can be accommodated in a single high-bay area served by an overhead crane, as shown in figure 1. The crane is essential for removal of roof beams from the cyclotron vault, and for dissembly of the magnet yokes. The adjacent shielded areas for isotope production and for radiotherapy can be housed in a low-bay section.

Controls and services will be close to the main cyclotron vault, while the experimental data-taking areas will be adjacent to the target rooms. Workshops, laboratories and offices will probably be located in a separate wing of the building. Additional wings should house the isotope-handling facility and radiotherapy patient-handling areas. Both ends of the main hall should then be free of outbuildings. In this way we hope to provide suitable facilities for a number of disciplines without restricting future expansion of the accelerator complex.

\section{References}

1. W.L. Rautenbach and A.H. Botha, Proposal for a South African National Accelerator Facility for Physics and Medicine, 7 th Int.Conf. on Cycl. and their Appl. (Birkhäuser, Basel-Stuttgart 1975) p. 117.

2. Technical Report of the Accelerator Task Group, National Accelerator Project, R.S.A. (PretoriaFaure, 1976)

3. R.W. Müller and R. Mart, Nucl. Instr. and Methods 86 (1970) 241.

4. H.N. Jungwirth et al., Design of Magnets for an OpenSector Cyclotron, Proc. 7th Int. Conf. on Cycl. and their Appl. (Birkhäuser, Basel-stuttgart 1975)p.193.

5. A.H. Botha and J.J. Kritzinger, Design of an RF System for an Open-Sector Cyclotron, ibid. p. 156.

6. W.P. Gertenbach, Atomic Energy Board Instrumentation Division Int. Report 1/TN/17/76 (Pel indaba 1976) 